Session 0458

\title{
Linux Workshop Session
}

\author{
Hugh Jack (jackh@gvsu.edu)
}

Grand Valley State University

\begin{abstract}
Linux is a free UNIX clone that was developed by volunteers around the world. Although Linux is almost a decade old, it went largely unnoticed by the general public until a couple of years ago. Since then it has become very popular with individual users, universities and large corporations. For example, IBM has made it a major part of their business strategy for server hardware. Many software companies already offer Linux versions of their software, including products such as Oracle, Labview and MSC Nastran. Other companies have developed embedded applications using Linux. Currently Linux can be found in devices as small as a wristwatch [1] and as large as a Beowulf class supercomputer [2]. The popularity of Linux is based on three factors:

- costs are lower because the software is free and it runs well on less expensive hardware.

- it has more software, capabilities, and features than other operating systems.

- the source code is open, so users can customize the operating system to meet their needs.

This workshop will present the Linux operating system in general, and its current status in computing. This will be followed by a discussion of the basic features and operation of a Linux system. User applications will also be discussed, and demonstrated. Finally the future of the operating system will be discussed. An outline of the presentation is given below.
\end{abstract}

\subsection{What is it?}

Linux is an open source operating system. It is open because users and developers can use the source code any way they want. This allows anyone to customize it, improve it and add desired features. As a result Linux is dynamic, evolving to respond to the desires and needs of the users. In contrast, closed operating systems are developed by a single corporation using static snapshots of market models and profit driven constraints.

Linux is free. This allows companies to use it without adding cost to products. It also allows people to trade it freely. And, with the profit motive gone, developers have a heightened sense of community interest. The Linux community has developed a tremendous spirit because of these core development concepts. 


\subsection{A (Brief) History}

Linux has existed since the early 1990s [3], but it grew out of previous developments in computing [4]. It was originally developed to be a Unix clone that would run on low cost computer hardware. Unix was developed in the 1970s. Through the 1970s and early 1980s it was used on large computers in companies and universities. During this time many refinements and enhancements were made. By the mid 1980s Unix was being used on many lower priced computers. By the end of the 1980s most universities were making use of Unix computers in computer science and engineering programs. This created a wealth of graduates who understood what they could expect from a mature operating system. But, it also created a demand to be able to do high level work at home on low priced machines.

Early in the 1990s Linux started as a project to create a Unix clone that would run on a personal computer. This project gained momentum quickly and by the mid 1990s it was ready for users. The first groups to adopt it were hobbyists, academics and internet services. At this time the general public was generally unaware of Linux but by the end of the 1990s it was beginning to enter the public sphere. By 2000 it had entered the popular press, and it was cited as a major threat to at least one existing operating system vendor. Now, it is available off-the-shelf in software and book stores.

$$
\begin{aligned}
\text { 1970s } & \text { - Unix developed at AT\&T labs } \\
1980 \text { s } & \text { - Unix became popular on high end computers } \\
& \text { - The Unix platform is refined and matures } \\
& \text { - Some versions of Unix were available for PCs - most notably QNX } \\
1990 s & \text { - Linus Torvalds begins working on a free Unix clone for PCs in } 1991 \\
& \text { - Others join the project it gets the name 'Linux' } \\
& \text { - By } 1993 \text { Linux begins to enter the mainstream of computer users } \\
& \text { - Linux machines constitute a large number of servers on the Internet } \\
& \text { - Many large companies begin to support Linux - e.g. Dell, IBM } \\
\text { 2000s } & \text { - Home and office users are supported with free office software } \\
& \text { - Linux is available in consumer products, such as Tivo recorders }
\end{aligned}
$$

\subsection{Hardware required and supported}

Modern computers have ample power for most computer applications. This is more true for Linux. At present there are versions of linux that will run on any platform from an IBM mainframe computer to a Palm Pilot. The smallest Linux installations can fit on a single floppy disk, and run on a diskless computer with a few MB of memory. On the other end of the spectrum, Linux will run on most high end computer systems. An average user would expect reasonable performance on a computer with an old Pentium 100 processor, $64 \mathrm{MB}$ of memory, and $2 \mathrm{~GB}$ of disk space. On newer computers the performance of the operating system is extremely fast. The list below gives some idea of the capabilities, but complete lists of supported hardware are available [5]. 
CPU - Intel family and clones, down to '386 processors

- Macintosh (Motorola)

- Others: Alpha, MIPS, Sparc, etc.

Memory- $16 \mathrm{MB}$ is a good minimum, $64 \mathrm{MB}$ is recommended

Disk - 200MB is a minimum, 2GB is recommended

Screen - Any size

Network- Any type

Others - Most PC hardware is supported - or will be soon

\subsection{Applications and uses}

By itself an operating system is somewhat useless, software applications are added to give desired functionality. Some of the common applications that a computer might be used for are listed below. Linux will support all of these applications, and more, with the right software [6].

Office - word processing, spreadsheets, etc.

Web and Internet Servers - host web sites

Server - databases and other institutional functions

Embedded - inside devices such as Tivo TV recorders

PDAs - an operating system for small handheld computers

Development - software authoring

\subsection{Advantages and disadvantages}

A partial list of advantages and disadvantages is given below. The cost, stability and open nature of the system have been winning over a large number of corporate adopters. But, adoption has been slowed by people who don't understand the nature of free software or have a perception that it is difficult to use. In some cases there are also some software packages that are not available for Linux, and won't run under simulators [22] - the most notable of these applications are first person shooting games.

Advantages:

Free - paying for it is optional

Open - the source code is available and can be changed

Goodwill - developers and users are very helpful

Faster - it doesn't require newer hardware, extra memory and larger disks

Stable - it is very uncommon for Linux to crash (no blue screens)

Flexibility - more capabilities and features

Complete - all of the software is available and open - no 'extra' software to buy

Security - very secure - unauthorized users can't change machine settings

Simplicity - point and click configuration

Disadvantages:

Compatibility - some programs will not run under simulators

Misunderstanding - some people believe 'you get what you pay for' 


\subsection{Getting it}

There are multiple distributions of Linux. While these all contain the Linux Kernel, they often include different pieces of software, and installation processes vary somewhat. The basic licensing agreement that Linux is distributed under requires that even if it is sold for a fee, it must be made available at no cost and it may also be copied freely by the user. As a result you can often download these distributions over the network at no cost [12][13]. The total download size can be up to $600 \mathrm{MB}$. An alternative is to buy a distribution (the typical cost is $\$ 30$ ) which includes a floppy disk, a CD-ROM and a brief manual. These can be found at any store that sells software. Sometimes the distribution will have a higher cost for 'deluxe' versions - this more costly package often includes telephone support.

\subsection{Distributions}

In total there are hundreds of Linux distributions. Many of these are specialized for features such as embedded systems, foreign languages, internet servers and security. The list below is for userfriendly installation and usage. The most successful of these distributions is Redhat. Some distributions, such as Mandrake, are based on the Redhat distribution, but with enhancements.

Redhat - the original consumer friendly Linux [7]

Mandrake - a Redhat derivative [8]

Caldera - another well established distribution [9]

Debian - a release that focuses on stability [10]

SuSe - yet another distribution [11]

\subsection{Installing}

Each distribution of Linux will have a slightly different installation procedure, but they all follow the basic steps below. The total time to install Linux will between one to two hours. Users with a high level of knowledge can opt to do advanced setup, and new users will have the option of letting the system suggest installation options.

1. Turn off the computer.

2. Insert a provided floppy disk (you can also boot from a CD on newer computers)

3. Turn the computer on, it will start to load Linux

4. You will be asked some questions about the type of installation you want

5. Linux will format the disks, and start to load the software

6. While it is loading you will be able to set times, dates and passwords

7. You be asked to set up the graphics for the window manager

8. When the installation is done the computer will reboot, and you will be ready to use it 


\subsection{Demonstration of Basic Features}

This section is a brief overview of the Linux operating system. The intention is to overview the basic components in the operating system. An administrator can manage the operating system using the graphical user interface (GUI), or using typed commands. New users often prefer to use the system using the GUI. Advanced users often prefer to use commands to administer the system, they are often faster and more reliable.

\subsection{Some Terminology}

The terms below are some of the keywords that are unique to Linux. These will appear during the installation, or during common usage of the system.

$\begin{array}{ll}\text { booting } & \begin{array}{l}\text { When a Linux computer starts it checks the hardware, and then starts } \\ \text { software. The process of booting takes less than a minute in most cases }\end{array} \\ \text { kernel } & \text { The core of the operating system that talks to all hardware and programs } \\ \text { shell } & \text { A windows that allows you to type commands } \\ \text { permissions } & \text { Control who can change what } \\ \text { GNU } & \text { (Gnu's Not Unix) A group that develops free software comprising a large } \\ \text { portion of Linux }\end{array}$

\subsection{X-Windows}

The GUI in Linux is actually two programs working together. The basic program is called $\mathrm{X}$ windows, and it provides basic connection to the screen, mouse, keyboard and sound card. The lookand-feel of the GUI is provided by the window manager. One simple window manager is called 'fvwm' and it can behave like Windows 95/98. Newer window managers include Gnome and KDE. While these both provide similar capabilities and features, most users develop personal preferences for a single window manager.

\subsection{File and directories}

The directory and file structure of Linux is hierarchical, much like other popular operating systems. The main directory for the system is call root and is indicated with a single slash ' $\%$. There are a number of subdirectories listed below that are used for storing system files, user files, temporary files and configuration files. A sample of the standard directories are shown below, and can be viewed with a file manager, or with keyboard commands. If other disks are used, such as a CDROM, or floppy disk, they are mounted under the root directory. (i.e., there are no 'C', 'A' or other drives, they are all under ' $\%$ ') 


$$
\begin{aligned}
& \text { /etc } \quad \text { - device and software configuration files are kept here } \\
& \text { /tmp } \quad \text { - temporary files are created here } \\
& \text { /home - user directories are kept here } \\
& \text { /var } \quad \text { - this is a place for log files, mail storage, etc. } \\
& \text { /usr } \quad \text { - software is installed under this directory } \\
& \text { /dev } \quad \text { - where devices are kept - they are accessed like files } \\
& \text { /bin } \quad \text { - some of the programs are kept in this directory }
\end{aligned}
$$

\subsection{Configuring}

Devices and settings can be configured under $\mathrm{X}$-windows using graphical tools. Settings can also be configured with text files, but this is not necessary. Examples of settings that the user or root might want to change are:

Modem properties for internet connection

Network card properties for connection to a LAN

Printer type and location

Customize the windows settings and behavior

Sound card settings and sounds for Window events

\subsection{User accounts and root}

Linux follows very strict conventions for file and directory permissions. These require that each file and directory be given specific permissions for public reading, writing and execution. Each user is given their own account with a password, so that access to the system is controlled. Only the root user can access all files and directories on the system. Other users are limited to files they own, or files that have been marked public. Typically the root user is only used for administration, and normal users use non-root accounts. This generally keeps the system safe from careless damage, and security breaches. Each user has their own home directory, normally in the '/home' directory. The permissions for files and directories are set so that the user has complete control over that directory.

\subsection{Security}

Security is not a significant problem for a computer that is not connected to a network, and passwords will protect it from 'honest thieves'. When connected to a network there is potential for security problems. These problems become more serious when the computer is connected to the network 24 hours a day. General rules to keep a computer safe (this applies to non-Linux computers also) are:

keep user passwords safe - these can be the start of a security breach protect the root password - loosing this throws the system wide open shut down unneeded programs - network programs sometime have bugs that open doors apply patches - software updates help close security holes 


\subsection{Desktop Tools}

Most users focus less on the Operating System, and more on the programs that it will run. The task list below includes many of the applications that would be desired by the average user. Most of the listed applications are free, with the exception of the games. Many of these packages are a standard part of Linux distributions.

- Office Software - these include spreadsheets, word processors, presentation software, drawing

Star Office [14] tools, database tools, 3D graphics tools

KOffice [15]

- File and Internet Browsers

Netscape - allows browsing of the internet [16]

Files - there are many file viewers that ease directory browsing

Eazel - allows active directory browsing [17]

- Administration and Utilities

Apache - the most popular web server program [18]

Postgres and MySQL - Database programs [19] [20]

Replace a microsoft networking server [21]

DOS/Windows Simulator VMWare [22]

- Entertainment

Audio and video

Tools (GIMP - similar to photoshop)

Games (Quake, Doom, SimCity)

\subsection{Future}

Linux use is growing quickly, and this is expected to increase. Within administrative circles Linux is now a proven commodity. IBM [1] has made Linux a core component of their business strategy. Home depot is adopting it for point-of-sale terminals. Mexico city is replacing their current operating system with Linux to redirect money to fighting poverty. In academic circles Linux has had a long history, and in many cases it has been adopted department wide in many computer science and some electrical engineering programs. 


\section{References}

[1] http://www.ibm.com

[2] http://www.beowulf.org, "The Beowulf Project".

[3] Hasan, R., "History of Linux", http://ragib.hypermart.net/linux.

[4] Polsson, K., "Chronology of Personal Computers”, http://www.islandnet.com/ kpolsson/complist

[5] http://www.linux.org/hardware/index.html, "Linux Friendly Hardware"

[6] http://www.linux.org/apps/index.html, "Applications"

[7] http://www.redhat.com

[8] http://www.mandrake.org

[9] http://www.caldera.com

[10] http://www.debian.com

[11] http://www.suse.com

[12] http://www.sunsite.unc.edu

[13] http://www.linux.com

[14] http://www.staroffice.com

[15] http://www.koffice.kde.org

[16] http://www.netscape.com

[17] http://www.eazel.com

[18] http://www.apache.org

[19] http://www.postgresql.org

[20] http://www.mysql.com

[21] http://www.samba.org

[22] http://www.vmware.com

\section{Hugh Jack}

Hugh Jack is an Assistant Professor in the Padnos School of Engineering at Grand Valley State University. He has been teaching there since 1996 in the areas of manufacturing and controls. His research areas include programmable logic controllers, process planning, robotics and rapids prototyping. He previously taught at Ryerson Polytechnic university for 3 years. He holds a Bachelors in Electrical Engineering, and Masters and Doctorate in Mechanical Engineering from the University of Western Ontario. 